\section{Precision Medical Treatment in Traditional Chinese Medicine}

\author{
Jian Chen ${ }^{1}$, Xue-Qing Hu${ }^{1}$, Qi-Long Chen ${ }^{1}$, Yuanjia $\mathrm{Hu}^{2}$ and \\ Shi-Bing Su${ }^{1 *}$
}

${ }^{1}$ Research Center for Traditional Chinese Medicine Complexity System, Shanghai University of Traditional Chinese Medicine, Shanghai, China

${ }^{2}$ State Key Laboratory of Quality Research in Chinese Medicine, Institute of Chinese Medical Sciences, University of Macau, Zhuhai, China

\begin{abstract}
Precision medical treatment in Traditional Chinese Medicine (TCM) refers to personalized medical diagnosis and treatment as well as precise prediction and prevention under the guidance of TCM theory, using technologies and methods of precision medicine. Here, we briefly introduced the accurate diagnosis and treatment based on ZHENG in TCM.

Keywords: Precision diagnosis; Precision treatment; Traditional Chinese Medicine; ZHENG
\end{abstract}

Precision medicine, based on personalized medicine, is to provide personalized and precise prediction, prevention, diagnosis and treatment by genomic, proteomic, metabolomic and other omics technologies and big data analysis techniques. Guided by the Traditional Chinese Medicine (TCM) theory, we may use the technologies and methods of precision medicine to analyze and mine the TCM "Four Diagnosis" information, to get better understanding of TCM ZHENG. Then we can make accurate ZHENG classification, diagnosis and carry out ZHENG based treatment. Precision medical treatment in TCM focuses on individual diagnosis and treatment in the views of system, like disease process and individual integrity with emphasis on main elements and primary contradictions in the system. The study of accurate diagnosis and treatment based on disease ZHENG is of great importance in TCM.

\section{Accurate Diagnosis of ZHENG}

ZHENG, also called TCM syndrome or TCM pattern, is an integral and essential part of TCM theory [1]. A TCM ZHENG, in essence, is a characteristic profile of all clinical manifestations collected by "Four Diagnosis" (observing, listening, asking and feeling), which can be identified by a TCM practitioner. Effective clinical treatments rely on the successful differentiation of a specific ZHENG [2].

\footnotetext{
*Corresponding author: Shi-Bing Su, Research Center for Traditional Chinese Medicine Complexity System, Shanghai University of Traditional Chinese Medicine, 1200 Cailun Road, Pudong, Shanghai 201203, China, Tel: +86
} 2151323013; E-mail: shibingsu07@163.com

Citation: Chen J, Hu X-Q, Chen Q-L, Hu Y, Su S-B (2017) Precision Medica Treatment in Traditional Chinese Medicine. J Altern Complement Integr Med 3: 024 .

Received: February 07, 2017; Accepted: February 27, 2017; Published: March 13, 2017
Accurate diagnosis is the basis of proper medical treatment. Objective and accurate identification of ZHENG is the premise of effective TCM treatment. The objectification of ZHENG and its essence discovery has always been the focus of TCM research. Accurate diagnosis of disease ZHENG is to objectively collect and analyze the clinical symptoms and signs with the application of system biology, bioinformatics and big data mining technologies etc. Then we may discover the biological material basis and biomarkers of ZHENG and make molecular classification of the phenotypes to explain its development and evolution mechanisms objectively and accurately.

Recently, some new technologies and methods such as the System Omics Approach were introduced in ZHENG research, which significantly facilitate the accurate diagnosis of ZHENG [2]. Previous studies have investigated ZHENG classification and biomarkers in chronic hepatitis B and liver cirrhosis, and have found that IL-10-819-C/T loci associated with the deficiency syndrome in hepatitis B caused cirrhosis [3]. Both miR-583 and miR-662 were able to distinguish the Liver and gallbladder damp heat syndrome from liver and kidney yin deficiency syndrome in patients with hepatitis B [4]. Additionally, the expression levels of PNP, AQP7 and PSMD2 were important to distinguish the damp heat syndrome from liver depression and spleen deficiency syndrome in hepatitis B caused cirrhosis [5]. These studies have provided the evidences for further study of ZHENG objectification to make accurate diagnosis of TCM.

\section{Accurate TCM Treatment}

Accurate TCM treatment is the individualized treatment using herbal medicine or acupuncture and moxibustion based on the accurate identification of ZHENG types, which depends on the different disease ZHENG molecular classification. Accurate treatment is fundamental to achieve desirable effect. Making diagnosis of disease via western medicine and combining the characteristics of TCM ZHENG with its molecular classification are beneficial to evaluating the curative effect of TCM treatment for disease ZHENG objectively and correctly and are also good to carry out the precise individualized treatment underlying ZHENG classification. Moreover, it may help to clarify curative effect mechanisms. Therefore, how to associate the genotyping of disease ZHENG with the effects of TCM treatment? This needs further study on the relationships between genetic variation or mutation and efficacy and safety of TCM treatment. Based on genotyping technologies of pharmacological genomics, previous study has that CYP1A2-G2964A is related to the curative effect of Fuzheng-Huayu tablet, a formula, which is able to improve the clinical symptoms of patients with the deficiency syndrome in hepatitis B caused cirrhosis [6]. Studies on transcriptional profiling and miRNA target network analysis have evidenced the efficacy of Fuzheng-Huayu [7]. This offers a case in point.

At present, the clinical research of ZHENG and ZHENG based treatment in TCM still lack large scale, multicenter clinical trial studies. Objective criterion for ZHENG classification and material basis for its formation and developing are important topics of the research in the future. The mode of precision treatment based on genes and molecular classification of disease ZHENG, may provide the useful 
research approaches and methods for TCM individualized treatment as well as the precision of TCM health care.

\section{References}

1. Su S-B, Lu A, Li S, Jia W (2012) Evidence-based ZHENG: a traditional Chinese medicine syndrome. Evid Based Complement Alternat Med 2012 246538.

2. Su S-B (2013) Recent advances in ZHENG differentiation research in traditional Chinese medicine. Int $\mathrm{J}$ integr med 1: 1-7.

3. Li Q-Y, Guo Z-Z, Liang J, Zhang W, Xu L-M, et al. (2012) Interleukin-10 genotype correlated to deficiency syndrome in hepatitis B cirrhosis. Evid Based Complement Alternat Med 2012: 298925.
4. Zhang H, Guan Y, Lu YY, Hu YY, Huang S, et al. (2013) Circulating miR-583 and miR-663 refer to ZHENG differentiation in chronic hepatitis B. Evid Based Complement Alternat Med 2013: 751341.

5. Lu Y-Y, Chen Q-L, Guan Y, Guo Z-Z, Zhang H, et al. (2014) Study of ZHENG differentiation in hepatitis $B$-caused cirrhosis: a transcriptional profiling analysis. BMC Complement Altern Med 14: 317.

6. Li QY, Guo ZZ, Deng X, Xu LM, Gao YQ, et al. (2013) Curative effects of ZHENG-Based Fuzheng-Huayu tablet on hepatitis $B$ caused cirrhosis related to CYP1A2 genetic polymorphism. Evid Based Complement Alternat Med 2013: 302131.

7. Chen Q, Wu F, Wan M, Dong S, Liu Y, et al. (2016) Transcriptional Profiling and miRNA-Target Network Analysis Identify Potential Biomarkers for Efficacy Evaluation of Fuzheng-Huayu Formula-Treated Hepatitis B Caused Liver Cirrhosis. Int J Mol Sci 17: 883. 